\title{
Distribution of Chitinolytic Enzyme in the Organs and Molecular Cloning of a Novel Chitinase Gene from the Kidney of Marbled Rockfish Sebastiscus marmoratus
}

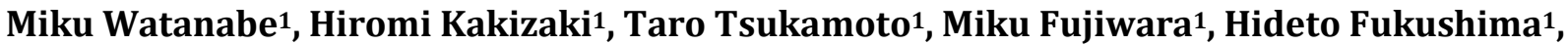 \\ Mitsuhiro Ueda' ${ }^{2}$, Masahiro Matsumiya ${ }^{1}$
}

\author{
${ }^{1}$ Department of Marine Science and Resources, College of Bioresource Sciences, Nihon University, Kanagawa, Japan \\ ${ }^{2}$ Department of Applied Biological Chemistry, College of Agriculture, Osaka Prefecture University, Osaka, Japan \\ Email: matsumiya@brs.nihon-u.ac.jp
}

How to cite this paper: Watanabe, M., Kakizaki, H., Tsukamoto, T., Fujiwara, M., Fukushima, H., Ueda, M. and Matsumiya, M. (2018) Distribution of Chitinolytic Enzyme in the Organs and Molecular Cloning of a Novel Chitinase Gene from the Kidney of Marbled Rockfish Sebastiscus marmoratus. Advances in Bioscience and Biotechnology, 9, 36-51.

https://doi.org/10.4236/abb.2018.91004

Received: December 25, 2017

Accepted: January 27, 2018

Published: January 30, 2018

Copyright $\odot 2018$ by authors and Scientific Research Publishing Inc. This work is licensed under the Creative Commons Attribution International License (CC BY 4.0).

http://creativecommons.org/licenses/by/4.0/

\begin{abstract}
Actinopterygii express two types of chitinase (acidic fish chitinase-1 (AFCase-1) and acidic fish chitinase-2 (AFCase-2)) that are active at acidic pHs and involved in digestion in the stomach. We proposed the existence of a new fish chitinase that has a non-digestive function. In this study, we used Sebastiscus marmoratus, for which characteristics and cDNA cloning of chitinase isozymes (SmChi-1, SmChi-2) in the stomach have been reported. Initially, we examined the distribution of chitinase and $\beta$ - $N$-acetylhexosaminidase (Hex) in the body and then we tried to clone novel chitinase cDNA from the kidney. Chitinase and Hex activities were measured using $p \mathrm{NP}-(\mathrm{GlcNAc})_{n},(n=$ $2,3)$ and $p$ NP-GlcNAc as substrates, respectively. Total RNA was extracted from the kidney. RT-PCR was performed to obtain chitinase cDNA fragments using reverse transcriptase with an oligo dT primer. The RACE method was used to obtain sequences of the upstream and downstream regions of cDNA. The full-length chitinase cDNA was determined using PrimeSTAR Max DNA polymerase with proofreading activity. High chitinase activity was observed in the stomach, as previously reported. In addition, relatively high activity was observed in the liver, spleen, kidney, and heart. In contrast, Hex activity was detected in all organs. This result is consistent with the report that Hex is related to body-wide metabolism. Full-length cDNA ( $S m C h i-3$ ) of the novel chitinase was obtained from the kidney, which contained 1440 bp open reading frames. The domain structure of SmChi-3 was assumed to be similar to those of SmChi-1 and SmChi-2. SmChi-1 and SmChi-2 have a serine and glycine-rich linker region, which is characteristic of AMCase. In contrast, Sm-
\end{abstract}


Chi-3 contained no apparent sequence in the linker region. Phylogenetic analysis revealed the existence of a new chitinase group, which was named fish chitinase-3 (FCase-3) and differed from AFCase-1 and AFCase-2.

\section{Keywords}

Chitinolytic Enzyme, Chitinase, Sebastiscus marmoratus, Distribution, cDNA Cloning

\section{Introduction}

Chitin, a $\beta$-1,4-linked aminopolysaccharide of $N$-acetyl-D-glucosamine (GlcNAc), is the second most abundant component of biomass in the world, after cellulose, and is found in the exoskeletons of arthropods, the cell walls of fungi, and the cuticles of nematodes [1] [2] [3]. In recent years, byproducts degraded from chitin have been shown to have various biological activities. For example, chitin oligosaccharides (GlcNAc) ${ }_{n}$, have been found to promote the growth of bifidobacteria, have immunostimulatory activity [4], and GlcNAc has been found to improve osteoarthritis [5] and dry skin [6].

Chitinolytic enzymes can be classified into the following two categories according to their degradation patterns: Endo-type chitinolytic enzymes, which degrade $\beta$-1,4-glycosidic bonds in a chitin polymer at random to produce chitin oligosaccharides $(\mathrm{GlcNAc})_{n}$ and are called chitinases (EC 3.2.1.14) [7] [8] [9]; and exo-type chitinolytic enzymes, which degrade a chitin polymer from the nonreducing end, one by one, to produce GlcNAc and are called $\beta$ - $N$-acetylhexosaminidase (Hex) (EC 3.2.1.52) [8] [9] [10]. Chitinases have been found in various organisms including mammals [11] [12] [13], fish [14] [15] [16] [17], mollusks [18] [19], insects [20] [21], plants [22] [23] [24], and fungi [25] [26] [27] and play important physiological roles in digestion [11] [14] [15] [16] [17], defense [12] [13] [22], aggression [18] [19], and ecdysis [18], in each of these organisms. Chitinases are classified into glycoside hydrolase (GH) families 18 and 19 on the basis of homology of amino acid sequences [27] and catalytic mechanisms [28] [29]. Family 18 chitinases are widely found in animals, plants, microorganisms, etc. [20]. Family 19 chitinases, on the other hand, are found primarily in higher plants and are reported to have strong antibacterial properties [20] [23].

We have conducted basic research about chitinases in several aquatic organisms. In the process of our research, we purified chitinase isozymes from fish stomachs, which are active at acidic pHs and are involved in digestion, and we investigated their properties [14] [15] [17]. We also have shown, through phylogenetic analysis based on amino acid sequences, which fish stomach chitinases form two fish-specific groups: acidic fish chitinase-1 (AFCase-1) and acidic fish chitinase-2 (AFCase-2) [30]. Furthermore, in fish, chitinase activity has been 
observed not only in the digestive tract, but also in other organs [31]. Also, an acidic mammalian chitinase (AMCase) was found in mammals, which are vertebrates like fish, and this AMCase is involved in digestion and absorption of food [11]. In addition, Chitotriosidase, a chitinase produced by macrophages that plays a role in defense against pathogens, was found in mammals [12] [13]. Based on these findings, we predicted that additional chitinases existed in fish and that these new chitinases would have physiological roles other than digestion, like their counterparts in mammals.

In this study, we used marbled rockfish Sebastiscus marmoratus, as a model species. Our group had previously purified three chitinase isozymes, SmChiA, SmChiB, and SmChiC from the stomach of $S$. marmoratus. These chitinases showed the optimum $\mathrm{pH}$ in the acidic region ( $\mathrm{pH} 2.0$ - 4.5). SmChiA and SmChiB preferentially degrades the second glycosidic bond from the non-reducing end of (GlcNAc) ${ }_{n}$ and SmChiC preferentially degrade the third glycosidic bond. SmChi-1 encoding SmChiA and SmChiB, and SmChi-2 encoding SmChiC were cloned. SmChi-1 and $S m C h i-2$ were classified into AFCase-1 and AFCase-2, respectively [14]. In this study, we measured chitinase activity and Hex activity to search for the presence of new chitinases in $S$. marmoratus using crude enzyme solutions of organs. Also, we examined the effect of $\mathrm{pH}$ on chitinase activity using crude enzymes prepared from the kidney and stomach. Furthermore, we tried to obtain full-length genes of a new chitinase, different from SmChi-1 and SmChi-2, from the kidney of $S$. marmoratus using RT-PCR methods. Finally, we conducted phylogenetic analyses with data obtained in this study and data of family 18 chitinases that have been entered in a database to clarify phylogenetic relations among fish chitinases.

\section{Materials and Methods}

\subsection{Material}

The marbled rockfish $S$. marmoratus was purchased from a fresh fish shop of Kanagawa ( $n=3$; average standard length $=25 \mathrm{~cm}$; average body weight $=275 \mathrm{~g})$. $p$-Nitrophenyl Di- $N$-Acetyl- $\beta$-chitobioside $\left(p \mathrm{NP}-(\mathrm{GlcNAc})_{2}\right)$ and $p$-Nitrophenyl Tri- $N$-Acetyl- $\beta$-chitobioside $\left(p \mathrm{NP}-(\mathrm{GlcNAc})_{3}\right)$ were purchased from Seikagaku corporation (Tokyo, Japan). $\quad p$-Nitrophenyl- $N$-Acetyl- $\beta$-D-glucosaminide ( $p$ NP-GlcNAc) was kindly supplied by Yaizu Suisankagaku Industry Co., Ltd. (Shizuoka, Japan).

\subsection{Measurement of Chitinolytic Enzyme Activity}

All organs $(0.5 \mathrm{~g})$ from $S$. marmoratus were homogenized in 3 volumes of 20 $\mathrm{mM}$ phosphate buffer $(\mathrm{pH} 7.3)$, and the homogenate was centrifuged at $9000 \times \mathrm{g}$ for $20 \mathrm{~min} 4^{\circ} \mathrm{C}$. Chitinase assays were conducted using the method of Ohtakara [26], with slight modification. The assays were completed using $p \mathrm{NP}-(\mathrm{GlcNAc})_{n}$, $(n=1-3)$ as the substrate. The crude enzyme solutions $(12.5 \mu \mathrm{l})$ and $5 \mu \mathrm{l}$ of 4 $\mathrm{mM} p \mathrm{NP}-(\mathrm{GlcNAc})_{n}$ were added to $12.5 \mu \mathrm{l}$ of $0.2 \mathrm{M}$ phosphate- $0.1 \mathrm{M}$ citrate 
buffer ( $\mathrm{pH}$ 6.0). The mixture was incubated at $37^{\circ} \mathrm{C}$ for $20 \mathrm{~min}$, and the reaction was stopped by adding $130 \mu \mathrm{l}$ of $0.2 \mathrm{M}$ sodium carbonate solution and the absorbance of the $p$-nitrophenol released was measured at $420 \mathrm{~nm}$ in a spectrophotometer. One unit of enzyme activity was defined as the amount of enzyme that was liberated by $1 \mu \mathrm{mol}$ of $p \mathrm{NP}$ per minute and was expressed as the activity per gram of the organs. The results are the mean of 3 replicates \pm standard deviation.

\subsection{Effect of $\mathrm{pH}$ on Chitinase Activity}

The effect of $\mathrm{pH}$ on chitinase activity was determined from the crude enzyme solutions obtained from the kidney and stomach of $S$. marmoratus. Chitinase activity was measured in a buffer solution with a $\mathrm{pH}$ between 2.0 to 8.0 by the method of distribution of chitinolytic enzymes activity, with $4 \mathrm{mM} p \mathrm{NP}-(\mathrm{GlcNAc})_{2}$ as the substrate.

\subsection{Molecular Cloning of a Novel Chitinase Gene from the Kidney}

Total RNA was extracted from the kidney of $S$. marmoratus using ISOGEN II (Nippon Gene, Tokyo, Japan), according to the manufacturer's instructions. Total RNA concentrations and purity were measured using the NanoVue spectrophotometer (GE Healthcare, Little Chalfont, UK). The first strand of cDNA was synthesized using $1.0 \mu \mathrm{g}$ total RNA and oligo dT primers (Table 1) with PrimeScript $^{\text {tom }}$ II Reverse Transcriptase (Takara Bio, Shiga, Japan) according to the manufacturer's instructions. For the reverse transcriptase-polymerase chain reaction (RT-PCR), three degenerate primers were designed from conserved sequences of the GH family 18 chitinases of vertebrates and internal sequence amplification was performed using GoTaq Green Master Mix (Promega, Madison, USA). The primers used in this study are shown in Table 1. The first PCR was performed using cDNA as a template, and $\mathrm{Chi} 3 \mathrm{~F} 1$ and $\mathrm{Chi} 3 \mathrm{R}$ as primers. The PCR parameters were 30 cycles of denaturation at $95^{\circ} \mathrm{C}$ for $30 \mathrm{~s}$, annealing at $50^{\circ} \mathrm{C}$ for $30 \mathrm{~s}$, and extension at $72^{\circ} \mathrm{C}$ for $30 \mathrm{~s}$. Nested PCR was performed using the products of the first PCR as templates, and Chi3 F2 and Chi3 R as primers, with the same PCR parameters as described above. cDNA fragments encoding the 3 'region of SmChi-3 were amplified using $S$. marmoratus cDNA as the template and the primer pairs Chi3 3'F1 and 3'RACE, and Chi3 3'F2 and 3'RACE. The PCR parameters were 30 cycles of denaturation at $95^{\circ} \mathrm{C}$ for $30 \mathrm{~s}$, annealing at $50^{\circ} \mathrm{C}$ for $30 \mathrm{~s}$, and extension at $72^{\circ} \mathrm{C}$ for $60 \mathrm{~s}$. The $5^{\prime} \mathrm{RACE}$ was performed using the 5 '-Full RACE Core Set (Takara Bio) according to the manufacturer's instructions. Full length $S m C h i-3$ was performed by PCR using specific primers (Chi3 full $\mathrm{F}$ and Chi3 full R) and PrimeSTAR ${ }^{\circ}$ Max DNA Polymerase (Takara Bio) with proofreading activity. The PCR products were resolved on a $2 \%$ agarose gel electrophoresis and purified using the Quantum Prep Freeze 'N Squeeze spin columns (Bio Rad, Hercules, USA). We subcloned all of the PCR products into pGEM-T Easy Vector (Promega) according to the manufacturer's instructions. 
Table 1. Primers used in this study.

\begin{tabular}{|c|c|c|c|c|}
\hline Primer name & Sequence $(5 '-3)$ & Length & Usage & $\begin{array}{l}\text { Annealing temperature } \\
\qquad\left({ }^{\circ} \mathrm{C}\right)\end{array}$ \\
\hline Oligo dT & CTGTGAATGCTGCGACTACGATTTTTTTTTTTTTTTTTTT & 40 mer & cDNA synthesis & \\
\hline Chi3 F1 (F) & TGYTAYTTYACNAAYTGG & 19 mer & Conserved region PCR & 50.0 \\
\hline Chi3 F2 (F) & GAYATHGAYTGGGARTAYCC & 19 mer & & \\
\hline Chi3 R (R) & TTCCARTARTTCATNGCRTARTC & 23 mer & & \\
\hline 3 RACE (R) & CTGTGAATGCGACTACGAT & 19 mer & 3'RACE PCR & 50.0 \\
\hline Chi3 3'F1 (F) & AAGAGGAAGTCCTCTGGAGG & 20 mer & & \\
\hline Chi3 3 'F2 (F) & GCTGAGGGAACAGCAACTGG & 20 mer & & \\
\hline Chi3 5'R1 (R) & GCAGCGGAGACCATTAATC & 19 mer & 5 'RACE PCR & 50.0 \\
\hline Chi3 5'R2 (R) & CCAGTTGCTGTTCCCTCA & 18 mer & & \\
\hline Chi3 5'F1 (F) & AGGAACCATTGATGCTGGTT & 20 mer & & \\
\hline Chi3 5'F2 (F) & TGGCAAAGTACCTGGACTT & 19 mer & & \\
\hline Chi3 5'F3 (F) & CGTGATGACGTATGACTTTCATG & 23 mer & & \\
\hline Chi3 5'P & GTGATGTCCTGTCACGCT & 18 mer & & \\
\hline Chi3 full $F(F)$ & GACATGACAAGAGACCATCCAA & 22 mer & Full length amplification & 55.0 \\
\hline Chi3 full R (R) & ACAGAGCATTTCAAACAGAGGG & 22 mer & & \\
\hline$\beta$-actin $(\mathrm{F})$ & GATCATGTTCGAGACCTTCAACAC & 24 mer & Organ expression & 50.0 \\
\hline$\beta$-actin (R) & TCCAATCCAGACAGAGTATTTAGC & 24 mer & & \\
\hline SmChi-1 (F) & ACTAGCGTGATCAAGTTCC & 19 mer & & \\
\hline SmChi-1 (R) & ACAGCTCCAATCGCGGA & 17 mer & & \\
\hline SmChi-2 (F) & CTCAGTCATTTAATTCCTGA & 20 mer & & \\
\hline SmChi-2 (R) & CCTGGCCAAGCTTGGGAAT & 19 mer & & \\
\hline SmChi-3 (F) & CAGTCTTCTGTCAACCTAC & 19 mer & & \\
\hline SmChi-3 (R) & GGTACTTTGCCATCTCTGC & 19 mer & & \\
\hline
\end{tabular}

The full-length amplification products of SmChi-3 carried out A-tailing, and were subcloned into pGEM-T Easy vector. Inserts were sequenced using the Big Dye Terminator Cycle Sequencing FS Ready Reaction Kit (Applied Biosystems, Waltham, USA).

\subsection{Phylogenetic Analysis of SmChi-3}

Phylogenetic analysis of chitinase from several organisms was carried out using the ClustalW2 program (EMBL-EBI: The European Bioinformatics Institute, European Molecular Biology Laboratory, Hinxton, England) and the tree view program. A bacterial chitinase (GenBank: X03657) was used as the outgroup.

\subsection{Organ-Specific Genes Expressions}

Total RNA was prepared for all organs from $S$. marmoratus. First-strand cDNA was synthesized using each total RNA $(0.5 \mu \mathrm{g})$ and an oligo dT primer, amplified using RT-PCR on the synthesized cDNA (1.0 $\mu \mathrm{g})$. SmChi-1, SmChi-2, and Sm- 
Chi-3, 250 bp gene fragments, were amplified using the first-strand cDNA as the template and the primer pairs $S m C h i-1$ (F) and $S m C h i-1$ (R) for $S m C h i-1, S m$ Chi-2 (F) and $S m C h i-2$ (R) for SmChi-2, and $S m C h i-3$ (F) and $S m C h i-3$ (R) for SmChi-3 (Table 1). To determine the amount of total RNA in each organ, $\beta$-actin mRNA fragments were amplified using specific primer pairs (Table 1). The PCR parameters were 30 cycles of denaturation at $95^{\circ} \mathrm{C}$ for $30 \mathrm{~s}$, annealing at $50^{\circ} \mathrm{C}$ for $30 \mathrm{~s}$, and extension at $72^{\circ} \mathrm{C}$ for $20 \mathrm{~s}$.

\section{Results and Discussion}

\subsection{Distribution of Chitinolytic Enzyme}

Measurement of activity of chitinolytic enzymes in organs of $S$. marmoratus (Figure 1) revealed that the chitinase activity assayed using $p \mathrm{NP}-(\mathrm{GlcNAc})_{2}$ and $p \mathrm{NP}-(\mathrm{GlcNAc})_{3}$ as substrates agreed with results that were reported previously [32], in which glycol chitin was used as a substrate and had the highest value in the stomach. In addition, relatively high activity also was observed in organs other than the digestive tract, including the liver, which is involved in metabolism and detoxification and contains much blood; the spleen, which is involved in blood production and storage; the kidney, which is involved in metabolism and hormone secretion; and the heart, which is involved in blood circulation. These

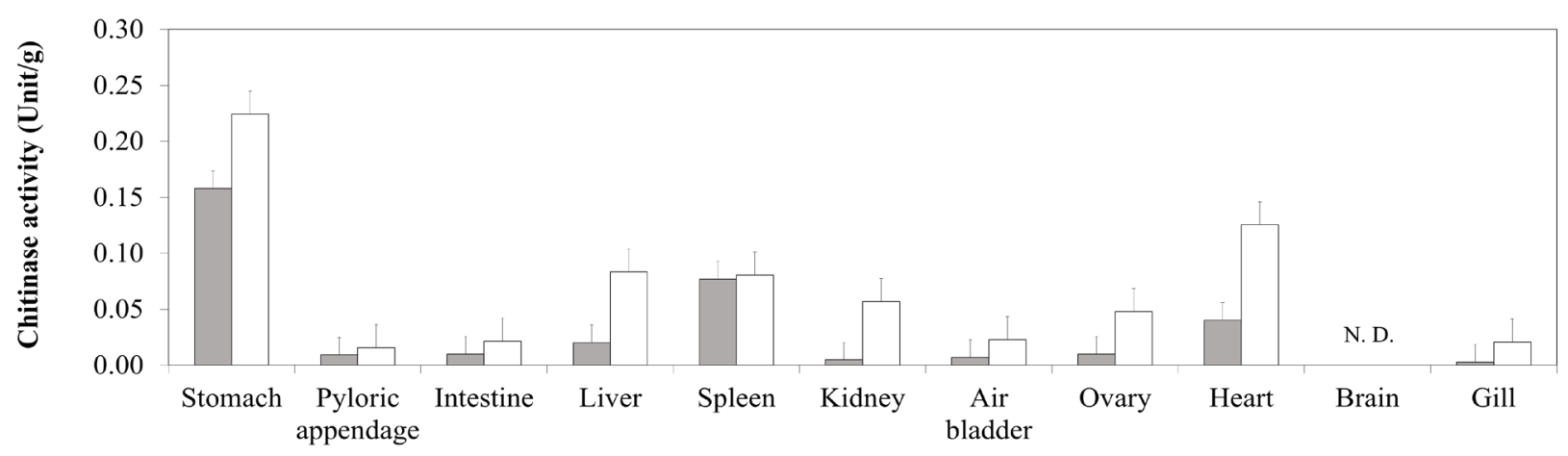

(a)

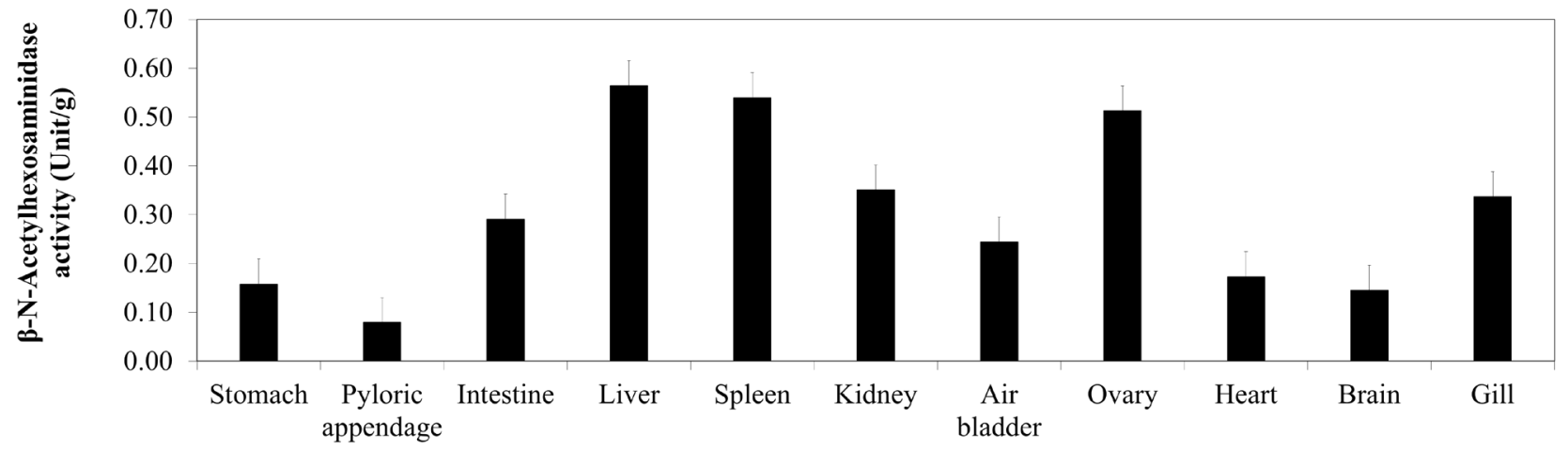

(b)

Figure 1. The distribution of the chitinolytic activities in the organs of $S$. marmoratus. (a): Chitinase activity, (b): $\beta$ - $N$-Acetylhexosaminidase activity, $\mathbf{\square}: p \mathrm{NP}-(\mathrm{GlcNAc}), \square: p \mathrm{NP}-(\mathrm{GlcNAc})_{2}, \square: p \mathrm{NP}-(\mathrm{GlcNAc})_{3}$. Bars represent the standard deviation $(n=3)$. 
findings suggested a possibility that chitinases in fish have physiological roles in several, non-digestive organs, as well as having a role in the digestion of ingested chitinous substances in the stomach. In addition, while the ratio of the degradation ability for $p \mathrm{NP}-(\mathrm{GlcNAc})_{2}$ to that for $p \mathrm{NP}-(\mathrm{GlcNAc})_{3}$ in the stomach was approximately $1: 1.5$, the ratio in the kidney was $1: 12.5$, which considerably differed from that in the stomach. This finding suggested the presence of chitinases in the kidney that degrade $p \mathrm{NP}-(\mathrm{GlcNAc})_{3}$ better than $p \mathrm{NP}-(\mathrm{GlcNAc})_{2}$, such as SmChiC in the stomach of $S$. marmoratus [14]. Hex activity measured at the same time had high values in the spleen, kidney, and heart. In fish, Hex activity in digestive organs has been thought to play a role in the degradation of $(\mathrm{GlcNAc})_{n}$ into GlcNAc, produced via the effect of chitinases in the stomach [31]. It has been reported that fish contain chitinous substances in their scales [33], have chitin synthases [34], and have a mechanism of carbohydrate metabolism [35]. From these findings, we thought that after ingested chitin was degraded to $(\mathrm{GlcNAc})_{n}$, by chitinases in the stomach, $S$. marmoratus would degrade (GlcNAc) ${ }_{n}$ into GlcNAc with chitinases and Hex, which would allow for the metabolism and use of (GlcNAc) ${ }_{n}$ that had been absorbed in the intestines and transferred to the bloodstream.

\subsection{Effect of pH on Chitinase Activity}

Chitinase activity of crude enzymes obtained from the stomach and kidney of $S$. marmoratus was measured at each $\mathrm{pH}$ using $p \mathrm{NP}-(\mathrm{GlcNAc})_{2}$ as a substrate (Figure 2). The stomach chitinase activity was greatest at $\mathrm{pH} 2.5$, but declined to $55 \%$ of the maximum at $\mathrm{pH} 5.0$, and to $15 \%$ at $\mathrm{pH}$ 7.0. In nature, the optimum $\mathrm{pH}$ for chitinase activity has been shown to be 2.5 , which was similar to that for chitinases obtained from the stomachs of fish including $S$. marmoratus [14] [15] [17] and to a property of AMCase [11]. On the other hand, the activity of the crude enzyme of the kidney peaked at $\mathrm{pH} 5.0$ and $40 \%$ of the maximum at $\mathrm{pH}$ 7.0. This was similar to a property of chitotriosidase, which for which the optimum $\mathrm{pH}$ is 5.0 [36]. The optimum $\mathrm{pH}$ for chitinase contained in the kidney was

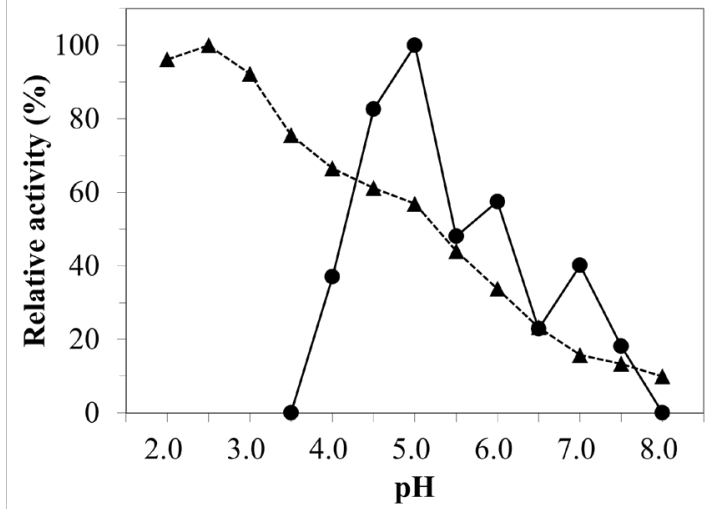

Figure 2. Effect of $\mathrm{pH}$ on chitinase activities in the kidney ( ) and stomach $(\mathbf{\Delta})$ of $S$. marmoratus. Substrate: $p \mathrm{NP}-(\mathrm{GlcNAc})_{2}$. 
slightly more neutral than the optimum $\mathrm{pH}$ of the stomach chitinase and this finding suggested that the chitinase activity detected in the kidney was attributable to the effect of a new chitinase that was different from the stomach chitinase. Chitinases have been obtained from fish organs other than those of the digestive system; for example, previous studies have reported the activity of chitinase in the blood serum of Salmo gairdneni [37] and a $75 \mathrm{kDa}$ chitinase obtained from the blood serum of freshwater, which has an optimum $\mathrm{pH}$ of 7.0 and considerable degradation capacity for $4 \mathrm{MU}(\mathrm{GlcNAc})_{2}$ rather than $4 \mathrm{MU}(\mathrm{GlcNAc})_{3}$, which was used as a substrate [38]. Although the kidneys of $S$. marmoratus are also non-digestive organs, the ability of the kidney chitinase of $S$. marmoratus to degrade substrates and the optimum $\mathrm{pH}$ differed from those of the chitinase of the plasma of Oreochromis niloticus. These results demonstrated the possibility that the chitinase of the kidney of $S$. marmoratus is a new chitinase isozyme different from chitinases contained in the blood serum of fish.

\subsection{Molecular Cloning of SmChi-3}

As a result of amplification of internal sequences of a gene of a new chitinase obtained from the kidney of $S$. marmoratus, 350 bp gene fragments were obtained. Analysis of base sequences of the gene fragments, conducted by the National Center for Biotechnology Information Basic Local Alignment Search Tool (NCBI Blast), showed 94\% homology with fChi3 of Paralichthys olivaceus. After this analysis, unknown regions of the upstream and downstream regions of the chitinase obtained from the kidneys of $S$. marmoratus were amplified using the RACE method. As a result, initiation codons and termination codons were found in the upstream regions and the downstream regions, respectively. Full-length genes of the chitinase obtained from the kidney of $S$. marmoratus were amplified using a sense primer and an antisense primer (designed with upstream and downstream untranslated regions (UTRs) in mind, respectively) and an enzyme with proofreading activity. As a result, a $1618 \mathrm{bp}$ full-length gene of the chitinase obtained from the kidney of $S$. marmoratus (SmChi-3) was obtained. This gene contained a $1440 \mathrm{bp}$ open reading frame (ORF) encoding 480 amino acids (Figure 3). The base sequence of SmChi-3 and some pieces of information were registered with the database of DDBJ and an accession number (LC077733) was issued.

Molecular weight of SmChi-3 was estimated at $51 \mathrm{kDa}$ based on its deduced amino acid sequence (SmChi-3) using the Compute pI/Mw tool of the Expert Protein Analysis System (ExPASy, Lausanne, Switzerland). This value is most similar to that of SmChi-1 (AB686658; $49 \mathrm{kDa})$, SmChi-2 (AB686659; $52 \mathrm{kDa})$, and chitotriosidase $(51 \mathrm{kDa})$ obtained from a mammal (Rattus norvegicus), which were estimated using the same method. This value was considerably lower than the value of $75 \mathrm{kDa}$ for the chitinase obtained from blood serum of $O$. niloticus [38]. Figure 4 shows SmChi-3, two chitinases obtained from the stomach of $S$. marmoratus (SmChi-1 and SmChi-2) [14], and the deduced amino acid 
G A C A T G A C A A G A G A C C A T C C A A A C G C A A C C A C C

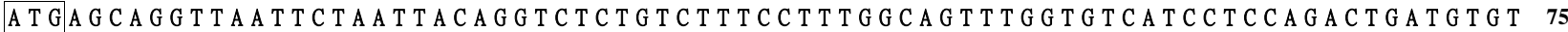

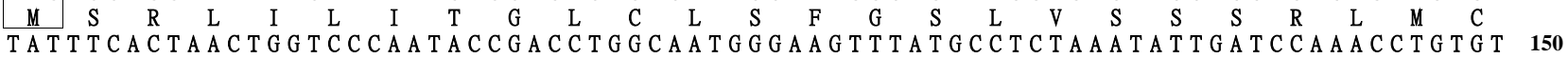

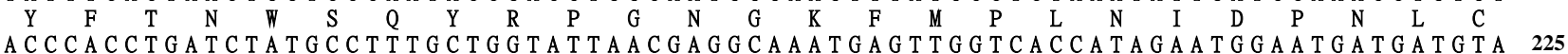

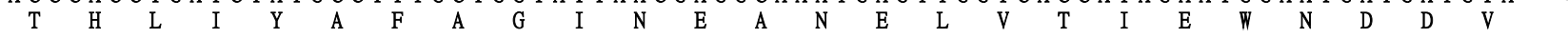

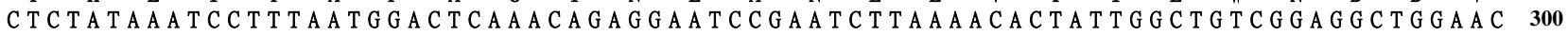

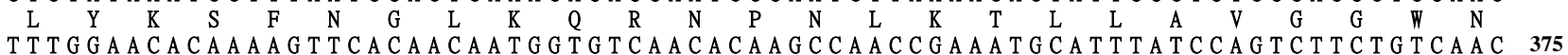

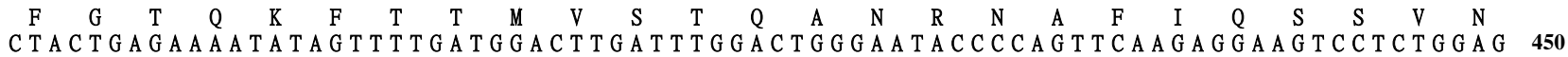

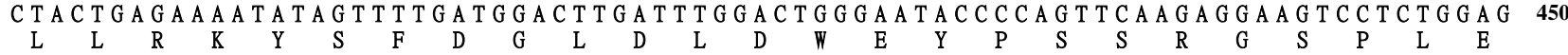

GACAAGCAGAGAT T CACGGTGTTATGCAAGGAACTCCTAGAGGCCTATCAGGCTGAGGGAACAGCAACTGCCGG 525

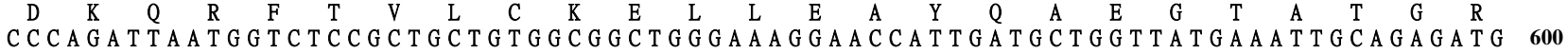

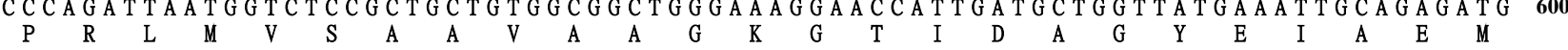

GCAAAGT ACCTGGACT T TATTAACGTGATGACGTATGACT T T CATGGCACCTGGGAGAGCGTGACAGACATCAC 675

$\begin{array}{ccccccccccccccc}A & K\end{array}$

$S$ P L Y N G S H D T G D H V T L N T D F A M R Y W

CGGGACAAGGGAACACCTGTAGAAAAGCTAAATATGGGCT T T GCTACATACGGGAGAGCAT TCCGACTGTCTACT 825

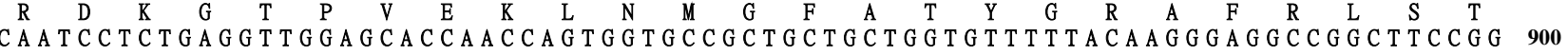

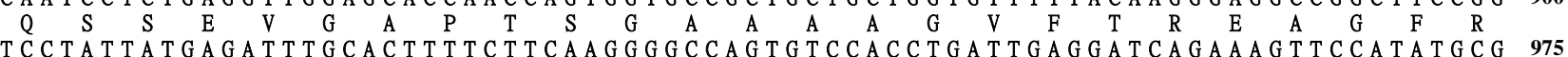

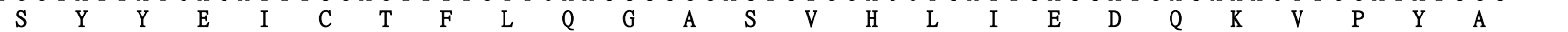

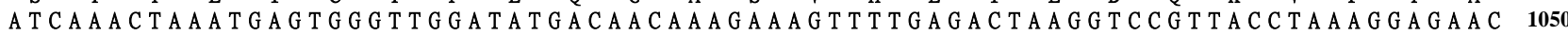

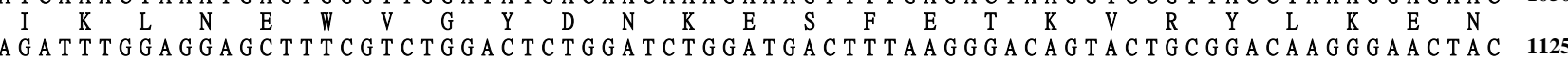

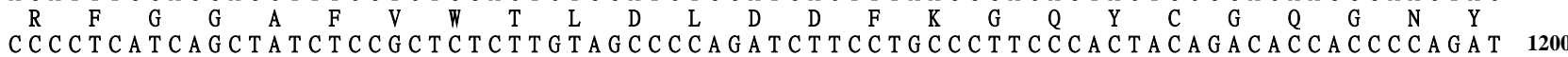

$\mathrm{C}$
$\mathrm{C}$

CAAGT GACTCCATCTACAAACATAGATCAACCTGACACCACATCTCGCCCCAGACCAGCAATCACTACT T CA T C C 1275

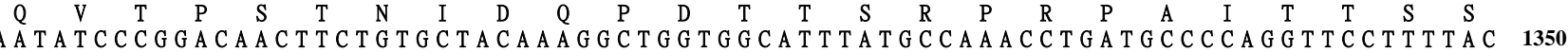

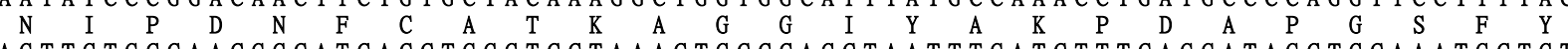

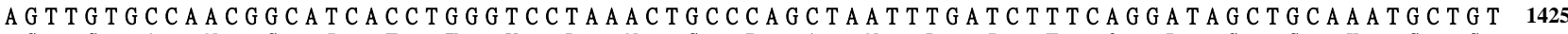

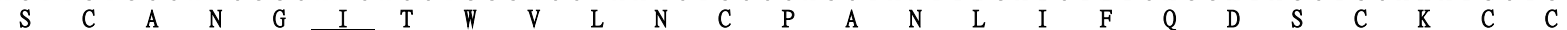

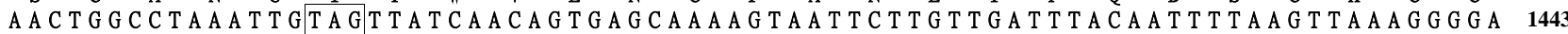
$\mathrm{N} W \mathrm{P}$ K $\mathrm{L}$,

C C T A T T A T G C T T A T T T T CA G G T G C A T A C T T C T T G T A T T T G G G C T T T T C T T T T C T C A T A C C G G C T G T G C T G CA G T A

C C T C T T T T C A

Figure 3. Deduced amino acid sequences and bases for SmChi-3 from the kidney of $S$. marmoratus. DDBJ accession No. LC077733.

sequences of genes, corresponding to Chi-3, of four varieties of fish and their predicted domain structures for comparison. SmChi-1, SmChi-2, and SmChi-3 consisted of N-terminal signal peptides, a catalytic domain, a chitinase insertion domain, a linker region, and a chitin binding domain, and the catalytic domain contained a sequence peculiar to the active site of GH family 18 chitinase, DXXDXDXE [39]. While SmChi-1 and SmChi-2, like AMCase [13], have repeated sequences of turn/coil-forming amino acids serine and glycine in the linker region, SmChi-3 does not have such characteristic repeated sequences. The same was true for fChi3 of P. olivaceus [16], CHIT3 of Scophthalmus maximus [40], and Chi-3 of Danio rerio [41]. Furthermore, comparison of Chi-3 of S. marmoratus, Hexagrammos otakii, Sardinops melanostictus, P. olivaceus [16], and Thunnus orientalis showed homology of $45 \%$ or more probability among the five varieties of fish. These results suggested the possibility that a Chi-3 gene ubiquitously exists in fish.

\subsection{Phylogenetic Analysis of SmChi-3}

Phylogenetic analysis of SmChi-3 was conducted on the basis of homology of deduced amino acid sequences of family 18 chitinases of other vertebrates and of 


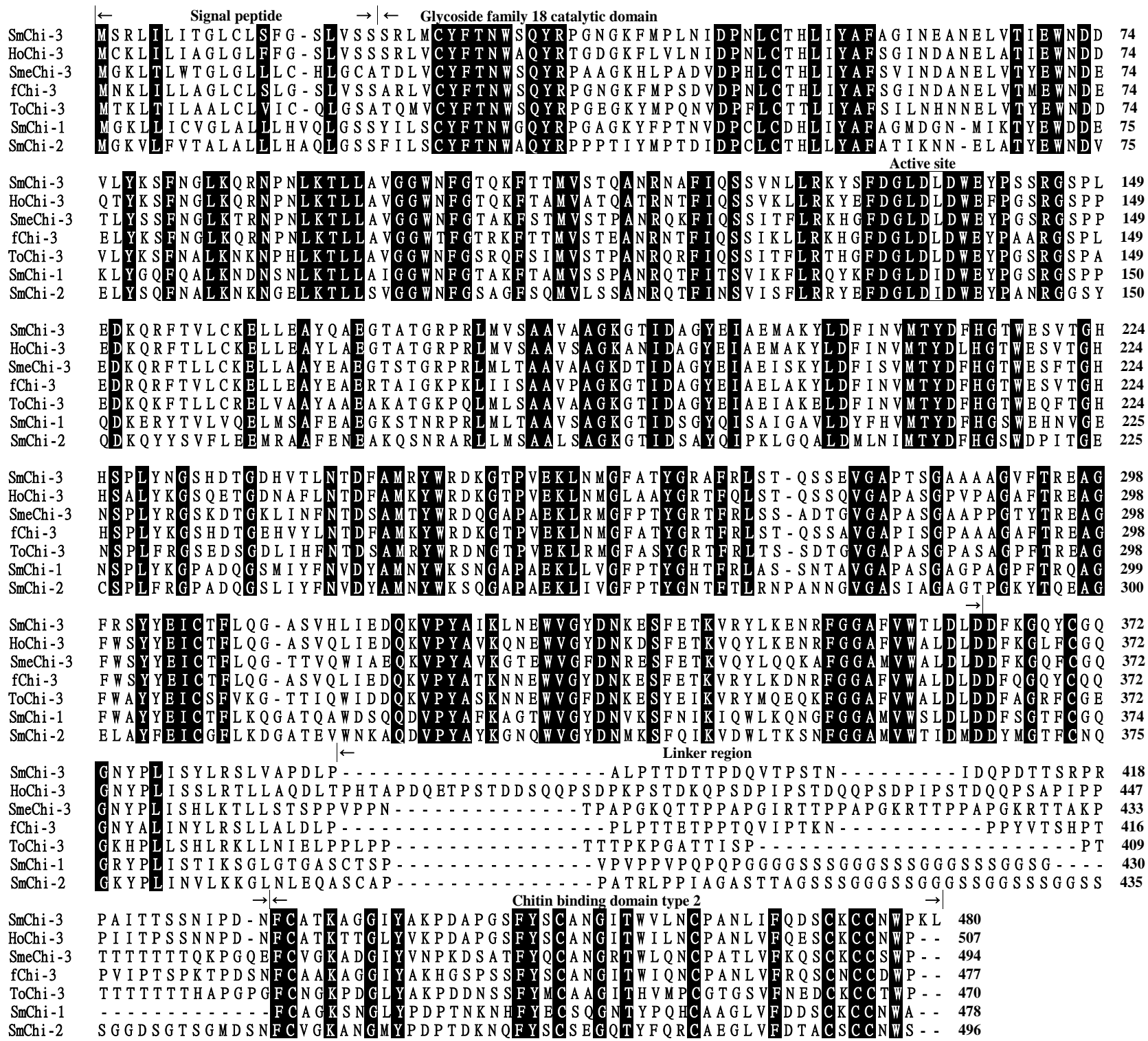

Figure 4. Alignment of amino acid sequences of SmChi-3 (S. marmoratus Chi-3), HoChi-3 (H. otakii Chi-3: DDBJ accession No. LC218726), SmeChi-3 (S. melanostictus Chi-3: DDBJ accession No. LC119087), fChi-3 (P. olivaceus Chi-3: DDBJ accession No. AB121734), ToChi-3 (T. orientalis Chi-3: DDBJ accession No. AB678426), SmChi-1 (S. marmoratus Chi-1: DDBJ accession No. AB686658), and SmChi-2 (S. marmoratus Chi-2: DDBJ accession No. AB686659).

chitinase from Serratia marcescens, which was used as an outgroup (Figure 5). As a result, like in previous work [14] [15] [31], chitinases expressed in the stomachs and lungs of mammals form an AMCase cluster, chitinases expressed in the tongue and spleen and derived from macrophages form Chitotriosidase clusters, and chitinases from the stomach of ray-finned fish form AFCase- 1 and AFCase- 2 clusters. However, SmChi-3 belonged neither to AFCase-1 cluster nor AFCase-2 cluster. SmChi-3 of $S$. marmoratus, the full-length gene sequence of which was obtained in this study, formed a new cluster that is obviously different from other chitinases, with the following chitinases registered in gene database under the various designations: SmeChi-3 of S. melanostictus, HoChi-3 of 


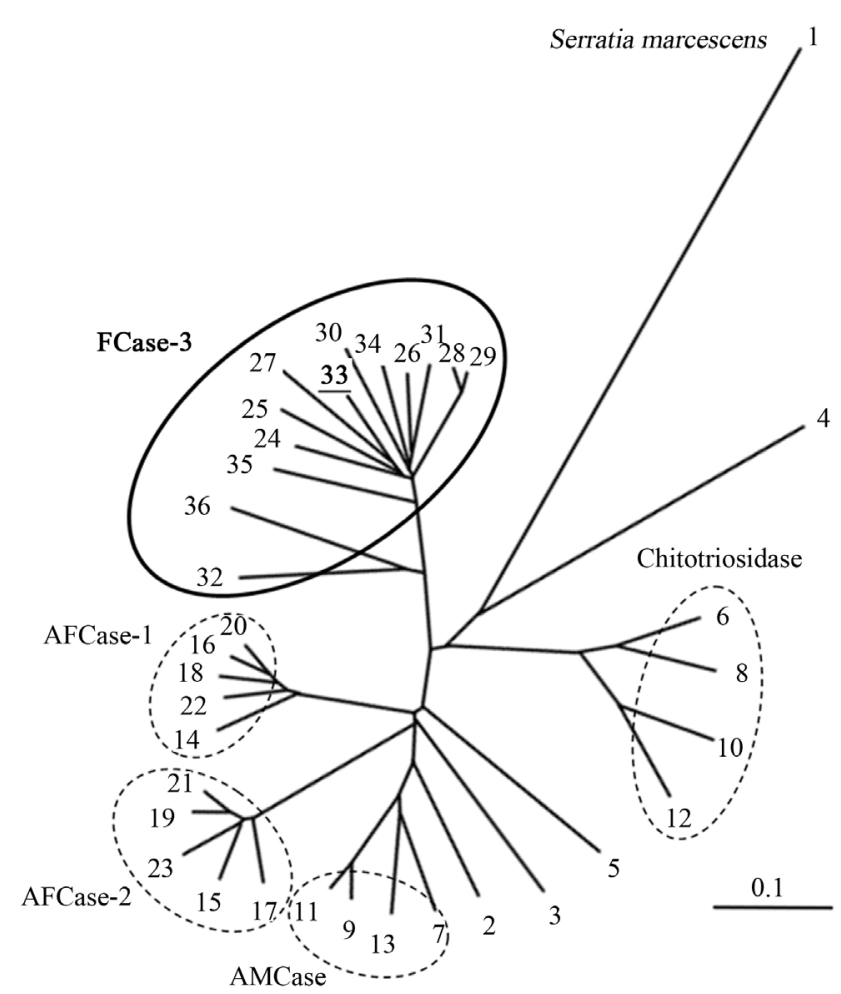

\begin{tabular}{|c|c|c|c|}
\hline No. & Scientific name & Information & Accession No. \\
\hline 1 & Serratia marcescens & Bacteria chitinase & $\mathrm{X} 03657$ \\
\hline 2 & Gallus gallus & Grandular stomach & AB071038 \\
\hline 3 & Latimeria chalumnae & Stomach & AB704869 \\
\hline 4 & Lethenteron japonicum & Liver & EU741679 \\
\hline 5 & Prionace glauca & stomach, PgChi & AB872008 \\
\hline 6 & Equus caballus & Chitotriosidase & EF694759 \\
\hline 7 & Homo sapiens & Stomach, Lung, AMCase & AF290004 \\
\hline 8 & Homo sapiens & Macrophage, Chitotriosidase & U29615 \\
\hline 9 & Mus musculus & Stomach, AMCase & EF094027 \\
\hline 10 & Mus musculus & Tongue, Chitotriosidase & AY458654 \\
\hline 11 & Rattus norvegicus & Stomach, AMCase & AY486074 \\
\hline 12 & Rattus norvegicus & Spleen, Chitotriosidase & DQ286232 \\
\hline 13 & Sarcophilus harrisii & AMCase & XM003769766 \\
\hline 14 & Epinephelus coioides & Chi1 & FJ169895 \\
\hline 15 & Epinephelus coioides & Chi2 & FJ169894 \\
\hline 16 & Paralichthys olivaceus & Stomach , fChi1 & AB121732 \\
\hline 17 & Paralichthys olivaseus & Stomach, fChi2 & AB121733 \\
\hline 18 & Parapristipoma trilineatum & Stomach, PtChi-1 & AB642677 \\
\hline 19 & Parapristipoma trilineatum & Stomach, PtChi-2 & AB642678 \\
\hline 20 & Pennahia argentata & Stomach, PaChi-1 & AB605774 \\
\hline 21 & Pennahia argentata & Stomach, PaChi-2 & AB605775 \\
\hline 22 & Sebastiscus marmoratus & Stomach, SmChi-1 & AB686658 \\
\hline 23 & Sebastiscus marmoratus & Stomach, SmChi-2 & AB686659 \\
\hline 24 & Labrus bergylta & Muscle, chitotriosidase-1-like & XP_020510835 \\
\hline 25 & Larimichthys crocea & AMCase & KQ040840 \\
\hline 26 & Lates calcarifer & Brain, Kidney, chitotriosidase-1-like & XP_018529975 \\
\hline 27 & Hexagrammos otakii & Kidney, HoChi-3 & LC218726 \\
\hline 28 & Neolamprologus brichardi & AMCase-like & XM_006794203 \\
\hline 29 & Oreochromis niloticus & blood, AMCase & XP_019205294 \\
\hline 30 & Oryzias latipes & chitotriosidase-1-like & XM_011477445 \\
\hline 31 & Paralichthys olivaceus & Pancreas, Chi3 & AB121734 \\
\hline 32 & Sardinops melanostictus & Kidney, SmeChi-3 & LC119087 \\
\hline 33 & Sebastiscus marmoratus & Kidney, SmChi-3 & LC077733 \\
\hline 34 & Stegastes partitus & chitotriosidase-1-like & XM_008278664 \\
\hline 35 & Takifugu rubripes & AMCase-like & XM_003963502 \\
\hline 36 & Thunnus orientalis & Whole body, Chi3 & AB678426 \\
\hline
\end{tabular}

Figure 5. Phylogenetic tree for chitinase amino acid sequences developed using the neighbor joining method in the ClustalW program. The right table lists the scientific name, information, and accession No. of chitinase used in the phylogenetic tree analysis. The numbers in the phylogenetic tree and table correspond to each other. A chitinase from bacteria was used as an outgroup. The scale bar indicates the substitution rate per residue.

H. otakii, AMCase of Larimichthys crocea, AMCase-like of Neolamprologus brichardi, chitotriosidase-1-like of Oryzias latipes, fChi3 of P. olivaceus, chitotriosidase-1-like of Stegastes partitus, AMCase-like of Takifugu rubripes, Chi3 of T. orientalis, chitotriosidase-1-like of Labrus bergylta (muscle) (XP_020510835), with AMCase of O. niloticus (blood) (XP_019205294), and chitotriosidase-1-like of Lates calcarifer (brain, kidney) (XP_018529975). One of the functions of chitinases in fish organs other than the stomach is said to be defense-related [16] [38] [40] [41] [42]. Examples are chitinases in blood plasma [38] [42] and chitinases on the surface of mucous membranes, which prevent attachment to and entry of pathogens [40]. Because these chitinase genes of various fish, which were grouped into this new cluster, are widely distributed in the body, including in the liver, kidneys, brain, muscle, and blood, it was suggested that the chitinase genes belonging to this cluster are present in fish regardless of fish variety and have various physiological roles besides digestion and defense. We named this new cluster of fish chitinase genes Fish Chitinase-3 (FCase-3). Furthermore, because Chi-3 of $S$. melanostictus and that of $T$. orientalis branch off in this phylogenetic analysis, the FCase-3 cluster may be divided further.

\subsection{Organ-Specific Genes Expressions}

Expression of SmChi-1, SmChi-2, SmChi-3, and $\beta$-actin, which is used as a house- 
keeping gene, in organs of $S$. marmoratus were analyzed by a semi-quantitative RT-PCR method and the result is shown in Figure 6. SmChi-1, classified into the AFCase-1 cluster, was expressed strongly in the stomach and heart and also expressed slightly in the pyloric appendage and ovary. SmChi-2, classified into the AFCase- 2 cluster, was expressed strongly in the stomach and also expressed slightly in the heart. In contrast, SmChi-3, classified into the FCase-3 cluster, was newly identified in this study and was expressed in the liver and kidney, where SmChi-1 and SmChi-2 were not expressed. fChil of P. olivaceus [16], PaChi-1 of P. argentata [31], and SmeChi-1 of $S$. melanostictus [15], which were classified into the AFCase-1 cluster in the phylogenetic analysis, were reported to be expressed strongly in the stomach and slightly in the gonad. fChi2 of $P$. olivaceus [16], PaChi-2 of $P$. argentata [31], and SmeChi-2 of S. melanostictus [15], which were classified into the AFCase-2 cluster were reported to be expressed strongly in the stomach. Distribution of mRNA of SmChi-1 and SmChi-2 was similar to that of fish classified into AFCase-1 and AFCase-2, respectively [15] [16] [31]. These findings indicated the possibility that AFCase-1 and AFCase-2 in fish have the same roles. In other words, SmChi-1 and SmChi-2 play roles in digestion and degradation of ingested chitin and chitin-containing organisms (which enter the stomach and pyloric appendage after initial entry into the digestive tract through the mouth), defense against chitin-containing external organisms in the gonad (ovary), and degradation of chitin oligosaccharides (GlcNAc) ${ }_{n}$ transferred to blood in the heart. On the other hand, because SmChi-3 was expressed only in the liver and kidney, SmChi-3 may be a new chitinase that plays physiological roles not only in digestion, but also in metabolism and carbohydrate storage.

\section{Conclusion}

In the body of $S$. marmoratus, relatively high chitinase activity was observed not only in the stomach, but also in the liver, spleen, kidney, and heart. Hex activity, on the other hand, was detected widely throughout the body; especially high Hex activity was observed in the spleen, kidney, and heart. Optimum $\mathrm{pH}$ for a chitinase contained in the crude enzyme of the stomach of $S$. marmoratus was

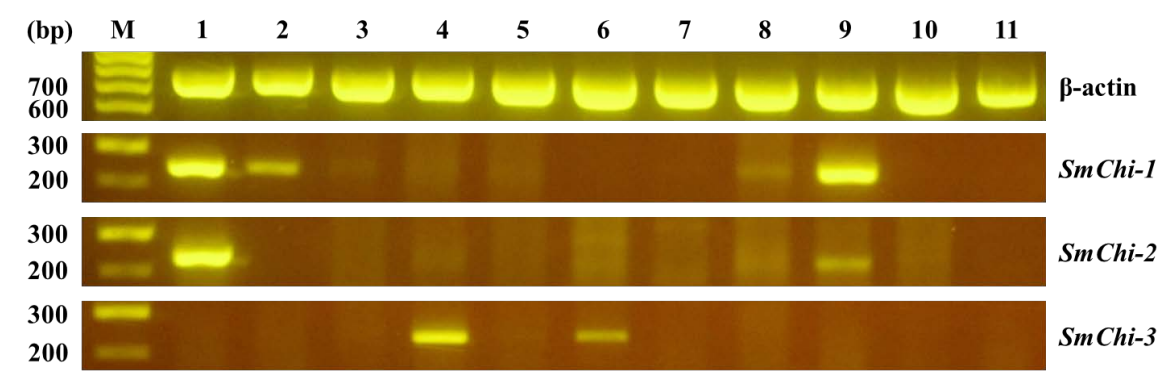

Figure 6. Expressions of $S m C h i-1, S m C h i-2, S m C h i-3$, and $\beta$-actin mRNA in organs of $S$. marmoratus by RT-PCR. Lane M: markers. Lane 1: stomach. Lane 2: pyloric appendage. Lane 3: intestine. Lane 4: liver. Lane 5: spleen. Lane 6: kidney. Lane 7: air bladder. Lane 8: ovary. Lane 9: heart. Lane 10: brain. Lane 11: gill. $\beta$-actin was used as a control. 
measured using $p \mathrm{NP}-(\mathrm{GlcNAc})_{2}$ as a substrate and was determined to be at $\mathrm{pH}$ 2.5. Its optimal $\mathrm{pH}$ was similar to that of previously reported fish stomach chitinases. On the other hand, the ratio of the degradation ability for $p \mathrm{NP}-(\mathrm{GlcNAc})_{2}$ to that for $p \mathrm{NP}-(\mathrm{GlcNAc})_{3}$ of a chitinase contained in the kidney and its optimum $\mathrm{pH}$ was different from those of previously reported chitinases obtained from the stomach of $S$. marmoratus and the blood plasma of $O$. niloticus. Molecular weight estimated from a deduced amino acid sequence of the new chitinase gene SmChi-3 (ORF, $1440 \mathrm{bp}$ ) obtained from the kidney of $S$. marmoratus in this study was $51 \mathrm{kDa}$, which was most similar to the molecular weight of chitotriosidase obtained from $R$. norvegicus $(50 \mathrm{kDa})$, but was remarkably lower than a chitinase obtained from the blood plasma of $O$. niloticus $(75 \mathrm{kDa})$. These findings suggested that SmChi-3 was a new chitinase that was distinct from stomach chitinases and blood plasma chitinases. Although SmChi-3 had a domain structure analogous to that previously reported for SmChi-1 and SmChi-2, SmChi-3 did not have SG repeated sequences in the linker region as observed in SmChi-1 and SmChi-2. Phylogenetic analysis revealed that SmChi-3 formed a peculiar cluster Fish Chitinase-3 (FCase-3), which is different from clusters of previously reported chitinases obtained from ray-finned fish (AFCase-1 and AFCase-2). Furthermore, because Chi-3 of T. orientalis and that of pilchard branch off in this phylogenetic analysis, the FCase-3 cluster may be divided further. Expression analysis of $S m C h i-1, S m C h i-2$, and $S m C h i-3$ in organs made by a semi-quantitative RT-PCR method showed that expression of $S m C h i-3$ in the liver and kidney, where $S m C h i-1$ and $S m C h i-2$ were not expressed. Furthermore, the results of chitinase activity measurement in the body of $S$. marmoratus and expression analysis of each gene suggested a possibility that chitinase activity in the kidney and liver was attributable to $S m C h i-3$, but chitinase activity in the spleen was attributable to another chitinase different from $S m C h i-1, S m$ Chi-2, and SmChi-3.

\section{Acknowledgements}

This work was supported in part by College of Bioresource science, Nihon University Grant (2017).

\section{References}

[1] Muzzarelli, R.A.A. (1977) Chitin. Pergamon Press, Oxford.

[2] Khoushab, F. and Yamabhai, M. (2010) Chitin Research Revisited. Marine Drugs, 8, 1988-2012. https://doi.org/10.3390/md8071988

[3] Rinaudo, M. (2006) Chitin and Chitosan: Properties and Applications. Progress in Polymer Science, 31, 603-632. https://doi.org/10.1016/j.progpolymsci.2006.06.001

[4] Mei, Y.X., Chen, H.X., Zhang, J., Zhang, X.D. and Liang, Y.X. (2013) Protective Effect of Chitooligosaccharides against Cyclophosphamide-Induced Immunosuppression in Mice. International Journal of Biological Macromolecules, 62, 330-335. https://doi.org/10.1016/j.ijbiomac.2013.09.038

[5] Kim, C., Shores, L., Guo, Q., Aly, A., Jeon, O.H., Kim, D.H., Bernstein, N., Bhatta- 
charya, R., Chae, J.J., Yarema, K.J. and Elisseeff, J.H. (2016) Electrospun Microfiber Scaffolds with Anti-Inflammatory Tributanoylated N-Acetyl-D-Glucosamine Promote Cartilage Regeneration. Tissue Engineering Part A, 22, 689-697. https://doi.org/10.1089/ten.tea.2015.0469

[6] Sayo, T., Sakai, S. and Inoue, S. (2004) Synergistic Effect of N-Acetylglucosamine and Retinoids on Hyaluronan Production in Human Keratinocytes. Skin Pharmacology and Physiology, 17, 77-83. https://doi.org/10.1159/000076017

[7] Dahiya, N., Tewari, R. and Hoondal, G.S. (2006) Biochemical Aspects of Chitinolytic Enzymes: A Review. Applied Microbiology and Biotechnology, 71, 773-782. https://doi.org/10.1007/s00253-005-0183-7

[8] Patil, R.S., Ghormade, V. and Deshpande, M.V. (2000) Chitinolytic Enzymes: An Exploration. Enzyme and Microbial Technology, 26, 473-483. https://doi.org/10.1016/S0141-0229(00)00134-4

[9] Rovertus, J.D. and Monzingo, F.A. (1999) The Structure and Action of Chitinases. EXS, 87, 125-135.

[10] Slamova, K., Bojarova, P., Petraskova, L. and Kren, V. (2010) $\beta$ - $N$-Acetylhexosaminidase: What's in a Name...? Biotechnology Advances, 28, 682-693. https://doi.org/10.1016/j.biotechadv.2010.04.004

[11] Muzzarelli, R.A.A., Boudrant, J., Meyer, D., Manno, N., Demarchis, M. and Paoletti, M.G. (2012) Current Views on Fungal Chitin/Chitosan, Human Chitinases, Food Preservation, Glucans, Pectins and Inulin: A Tribute to Henribraconnot, Precursor of the Carbohydrate Polymers Science, on the Chitin Bicentennial. Carbohydrate Polymers, 87, 995-1012. https://doi.org/10.1016/j.carbpol.2011.09.063

[12] Boot, R.G., Renkema, H., Strijland, A., Zonneveld, A.J.V. and Aerts, J.M.F.G. (1995) Cloning of a cDNA Encoding Chitotriosidase, a Human Chitinase Produced by Macrophages. The Journal of Biological Chemistry, 270, 26252-26256. https://doi.org/10.1074/jbc.270.44.26252

[13] Boot, R.G., Blommaart, E.F.C., Swart, E., Vlugt, K.G.V.D., Bijl, N., Moe, C., Place, A. and Aerts, J.M.F.G. (2001) Identification of a Novel Acidic Mammalian Chitinase Distinct from Chitotriosidase. The Journal of Biological Chemistry, 276, 6770-6778. https://doi.org/10.1074/jbc.M009886200

[14] Ikeda, M., Shirase, D., Sato, T., Ueda, M., Hirabayashi, S. and Matsumiya, M. (2014) Primary Structure and Enzymatic Properties of Chitinase Isozymes Purification from the Stomach of the Marbled Rockfish Sebastiscus marmoratus. Journal of Chitin and Chitosan Science, 2, 106-116. https://doi.org/10.1166/jcc.2014.1048

[15] Kawashima, S., Ikehata, H., Tada, C., Ogino, T., Kakizaki, H., Ikeda, M., Fukushima, H. and Matsumiya, M. (2016) Stomach Chitinase from Japanese Sardine Sardinops melanostictus: Purification, Characterization, and Molecular Cloning of Chitinase Isozymes with a Long Linker. Marine Drugs, 14, 22.

https://doi.org/10.3390/md14010022

[16] Kurokawa, T., Tuji, S. and Suzuki, T. (2004) Molecular Cloning of Multiple Chitinase Genes in Japanese Flounder, Paralichthys olivaceus. Comparative Biochemistry and Physiology_Part B: Biochemistry \& Molecular Biology, 138, 255-268. https://doi.org/10.1016/j.cbpc.2004.03.015

[17] Matsumiya, M., Arakane, Y., Haga, A., Muthukrishnan, S. and Kramer, K.J. (2006) Substrate Specificity of Chitinase from Two Species of Fish, Greenling, Hexagrammos otakii, and Common Mackerel, Scomber japonicus, and the Insect, Tobacco Hornworm, Manducasexta. Bioscience, Biotechnology, and Biochemistry, 70, 971-979. https://doi.org/10.1271/bbb.70.971

[18] Gooday, G.W. (1999) Aggressive and Defensive Roles for Chitinases. EXS, 87, 
157-169. https://doi.org/10.1007/978-3-0348-8757-1_11

[19] Ogino, T., Tabata, H., Ikeda, M., Kakizaki, H. and Matsumiya, M. (2014) Purification of a Chitinase from the Posterior Salivary Gland of Common Octopus Octopus vulgaris and Its Properties. Journal of Chitin and Chitosan Science, 2, 135-142. https://doi.org/10.1166/jcc.2014.1049

[20] Adrangi, S. and Faramarzi, M.A. (2013) From Bacteria to Human: A Journey into the World of Chitinases. Biotechnology Advances, 31, 1786-1795. https://doi.org/10.1016/j.biotechadv.2013.09.012

[21] Merzendorfer, H. and Zimoch, L. (2003) Chitin Metabolism in Insects: Structure, Function and Regulation of Chitin Synthases and Chitinases. Journal of Experimental Biology, 206, 4393-4412. https://doi.org/10.1242/jeb.00709

[22] Ahmed, N.U., Park, J.I., Jung, H.J., Kang, K.K., Hur, Y., Lim, Y.P. and Nou, I.S. (2012) Molecular Characterization of Stress Resistance-Related Chitinase Genes of Brassica rapa. Plant Physiology and Biochemistry, 58, 106-115. https://doi.org/10.1016/j.plaphy.2012.06.015

[23] Karasuda, S., Tanaka, S., Kagihara, H., Yamamoto, Y. and Koga, D. (2003) Plant Chitinase as a Possible Biocontrol Agent for Use Instead of Chemical Fungicides. Bioscience, Biotechnology, and Biochemistry, 67, 221-224. https://doi.org/10.1271/bbb.67.221

[24] Wang, S., Shao, B., Fu, H. and Rao, P. (2009) Isolation of a Thermostable Legume Chitinase and Study on the Antifungal Activity. Applied Microbiology and Biotechnology, 85, 313-321. https://doi.org/10.1007/s00253-009-2074-9

[25] Karthik, N., Akanksha, K., Binod, P. and Pandey, A. (2014) Production, Purification and Properties of Fungal Chitinase-A Review. Indian Journal of Experimental Biology, 52, 1025-1035

[26] Ohtakara, A. (1988) Chitinase and $\beta$ - $N$-Acetylhexosaminidase from Pycnoporu cinnabarinus. Biomass Part B: Lignin, Pectin, and Chitin, 161, 462-470. https://doi.org/10.1016/0076-6879(88)61059-7

[27] Henrissat, B. and Bairoch, A. (1993) New Families in the Classification of Glycosyl Hydrolases Based on Amino acid Sequence Similarities. The Journal of Biochemistry, 293, 781-788. https://doi.org/10.1042/bj2930781

[28] Fukamizo, T., Koga, D. and Goto, S. (1995) Comparative Biochemistry of Chitinases-Anomeric Form of the Reaction Products. Bioscience, Biotechnology, and Biochemistry, 59, 311-313. https://doi.org/10.1271/bbb.59.311

[29] Koga, D., Yoshioka, T. and Arakane, Y. (1998) HPLC Analysis of Anomeric Formation and Cleavage Pattern by Chitinolytic Enzyme. Bioscience, Biotechnology, and Biochemistry, 62, 1643-1646. https://doi.org/10.1271/bbb.62.1643

[30] Ikeda, M., Kakizaki, H. and Matsumiya, M. (2017) Biochemistry of Fish Stomach Chitinase. International Journal of Biological Macromolecules, 104, 1672-1681. https://doi.org/10.1016/j.ijbiomac.2017.03.118

[31] Kakizaki, H., Ikeda, M., Fukushima, H. and Matsumiya, M. (2015) Distribution of Chitinolytic Enzymes in the Organs and cDNA Cloning of Chitinase Isozymes from the Stomach of Two Species of Fish, Chub Mackerel (Scomber japonicus) and Silver Croaker (Pennahia argentata). Open Journal of Marine Science, 5, 398-411. https://doi.org/10.4236/ojms.2015.54032

[32] Matsumiya, M. and Mochizuki, A. (1996) Distribution of Chitinase and $\beta$ - $N$-Acerylhexosaminidase in the Organs of Several Fishes. Fisheries Science, 62, 150-151. https://doi.org/10.2331/fishsci.62.150 
[33] Kumari, S. and Rath, P.K. (2014) Extraction and Characterization of Chitin and Chitosan from (Labeo rohit) Fish Scales. Procedia Materials Science, 6, 482-489. https://doi.org/10.1016/j.mspro.2014.07.062

[34] Wagner, G.P., Lo, J., Laine, R. and Almeder, M. (1993) Chitin in the Epidermal Cuticle of a Vertebrate (Paralipophrys trigloides, Blenniidae, Teleostei). Experientia, 49, 317-319. https://doi.org/10.1007/BF01923410

[35] Hemre, G.I., Mommsen, T.P. and Krogdahl, A. (2002) Carbohydrates in Fish Nutrition: Effects on Growth, Glucose Metabolism and Hepatic Enzymes. Aquaculture Nutrition, 8, 175-194. https://doi.org/10.1046/j.1365-2095.2002.00200.x

[36] Xiao, H.C. and Guo, P.C. (2008) Molecular Cloning and Characterization of Rat Chitotriosidase. DNA Sequence, 19, 121-129.

https://doi.org/10.1080/10425170701447499

[37] Lindsay, G.J.H., Walton, M.J., Adron, J.W., Hetcher, T.C., Cho, C.Y. and Cowey, C.B. (1984) The Growth of Rainbow Trout (Salmo gairdneni) Given Diets Containing Chitin and Its Relationship to Chitinolytic Enzymes and Chitin Digestibility. Aquaculture, 37, 315-324. https://doi.org/10.1016/0044-8486(84)90297-7

[38] Molinari, L.M., Pedroso, R.B., Scoaris, D.O., Ueda-Nakamura, T., Nakamura, C.V. and Filho, B.P.D. (2007) Identification and Partial Characterization of a Chitinase from Nile Tilapia, Oreochromis niloticus. Comparative Biochemistry and Physiology_Part B: Biochemistry \& Molecular Biology, 146, 81-87. https://doi.org/10.1016/j.cbpb.2006.09.004

[39] van Aalten, D.M., Synstad, B., Brurberg, M.B., Hough, E., Riise, B.W., Eijsink, V.G. and Wierenga, R.K. (2000) Structure of a Two-Domain Chitotriosidase from Serratia marcescens at 1.9-A Resolution. Proceedings of the National Academy of Sciences, 97, 5842-5847. https://doi.org/10.1073/pnas.97.11.5842

[40] Gao, C., Cai, X., Zhang, Y., Su, B., Song, H. and Li, C. (2017) Characterization and Expression Analysis of Chitinase Genes (CHIT1, CHIT2 and CHIT3) in Turbot (Scophthalmus maximus L.) Following Bacterial Challenge. Fish and Shellfish Immunology, 64, 357-366. https://doi.org/10.1016/j.fsi.2017.03.019

[41] Zinan, T., Chen, S., Shousheng, L., Hongmiao, W. and Shicui, Z. (2014) Functional Characterization of Chitinase-3 Reveals Involvement of Chitinases in Early Embryo Immunity in Zebrafish. Developmental \& Comparative Immunology, 46, 489-498. https://doi.org/10.1016/j.dci.2014.06.008

[42] Fange, R., Lundblad, G. and Lind, J. (1976) Lysozyme and Chitinase in Blood and Lymphomyeloid Tissues of Marine Fish. Marine Biology, 36, 277-282. https://doi.org/10.1007/BF00389289 